\title{
Chiral Recognition of Diketopiperazine Cyclo(Pro-Gly) and Propranolol Using (-)-Epigallocatechin-3-O-gallate
}

\author{
Takashi Ishizu,* Hiroyuki Tsutsumi, Emi Yokoyama, Haruka Tanabe, and Aoi Yokoyama \\ Faculty of Pharmacy and Pharmaceutical Sciences, Fukuyama University; Sanzo Gakuen-cho 1, Fukuyama, \\ Hiroshima 729-0292, Japan. \\ Received September 15, 2015; accepted November 16, 2015
}

In the ${ }^{1} \mathrm{H}-\mathrm{NMR}$ spectrum of a solution containing an equimolecular amount of cyclo(L-Pro-Gly), cyclo(D-Pro-Gly) and (-)-epigallocatechin-3-O-gallate (EGCg) in a $\mathrm{D}_{2} \mathrm{O}$, a difference in the chemical shift of ${ }^{1} \mathrm{H}-\mathrm{NMR}$ signal for $\mathrm{H}_{7 \alpha}, \mathrm{H}_{7 \beta, 8 \alpha}$ of the Pro residue was observed. Judging from the crystal structures of the $2: 2$ complexes of EGCg and cyclo(L-Pro-Gly), cyclo(D-Pro-Gly), the difference in the chemical shift resulted mainly from a magnetic anisotropic shielding effect by the ring current from the B ring of EGCg. Therefore, it was considered that chirality of cyclo(Pro-Gly) was recognized by EGCg in the $\mathrm{D}_{2} \mathrm{O}$ solution. Furthermore, in the ${ }^{1} \mathrm{H}$-NMR spectrum of a solution containing an equimolecular amount of racemic propranolol $((R)-$ and $(S)$-propranolols) and EGCg in $\mathrm{D}_{2} \mathrm{O}$, the ${ }^{1} \mathrm{H}$-NMR signal for $\mathrm{H}_{2}$ of the naphthalene group was observed as two doublets, suggesting that the racemic propranolol formed diastereomers of complexes with EGCg; as a result, chirality of propranolol was recognized by EGCg in the $\mathrm{D}_{2} \mathrm{O}$ solution.

Key words cyclo(Pro-Gly); propranolol; ${ }^{1} \mathrm{H}-\mathrm{NMR}$ spectrum; X-ray crystallographic analysis; (-)-epigallocatechin-3-O-gallate

When a hot tea beverage cools down, it becomes turbid and brown-white particles settle out. This phenomenon is called "creaming" or "a creaming-down reaction." Previously, Ina and colleagues reported that creaming down eventually occurs when an aqueous caffeine solution is poured into an aqueous solution of gallated catechin (-)-epigallocatechin-3-O-gallate (EGCg), which is most abundant in tea catechins. ${ }^{1)}$

Then, we attempted crystallization of the precipitate formed by the creaming-down reaction made from an aqueous solution of tea gallated catechin EGCg and caffeine, and as a result obtained a crystal which was determined to be a $2: 2$ complex of EGCg and caffeine by X-ray crystallographic analysis $^{2)}$ (Fig. 1a).

As shown in Fig. 1b, the caffeine moieties of the $2: 2$ complex were located in the space surrounding the top and lower walls of the $\mathrm{B}^{\prime}$ rings of EGCg moieties and right and left walls of the $\mathrm{A}$ and $\mathrm{B}$ rings of EGCg moieties. As a result, caffeine molecules were captured by the hydrophobic space formed by the three aromatic $\mathrm{A}, \mathrm{B}, \mathrm{B}^{\prime}$ rings of the $\mathrm{EGCg}$ in the $2: 2$ complex. Water molecules existed outside the space formed by the three aromatic $\mathrm{A}, \mathrm{B}, \mathrm{B}^{\prime}$ rings of $\mathrm{EGCg}$ and were not observed in the space, suggesting that the space had high hydrophobicity. It was therefore thought that the sticky precipitate was formed by creaming precipitated from the aqueous solution of EGCg and caffeine due to its high hydrophobicity.

The hydrophobic space is available for capture in various compounds in substitution for caffeine. Furthermore, it was assumed that the space formed by the three aromatic A, B, $\mathrm{B}^{\prime}$ rings of $\mathrm{EGCg}$ could recognize the chirality of compounds included in the space because the $\mathrm{C}$ ring of EGCg has two chiral carbon atoms, $\mathrm{C}_{2}$ and $\mathrm{C}_{3}$, and the hydrophobic space formed by the three aromatic $\mathrm{A}, \mathrm{B}, \mathrm{B}^{\prime}$ rings of $\mathrm{EGCg}$ was a chiral space. Many medicines are currently used in racemic form; however, it is desirable to use a single enantiomer from the viewpoint of adverse effects and pharmacokinetics. EGCg, which easily forms a complex with various compounds and dissociates in an aqueous solution, may be available as a new optical resolving agent for natural products and medicines. (a)

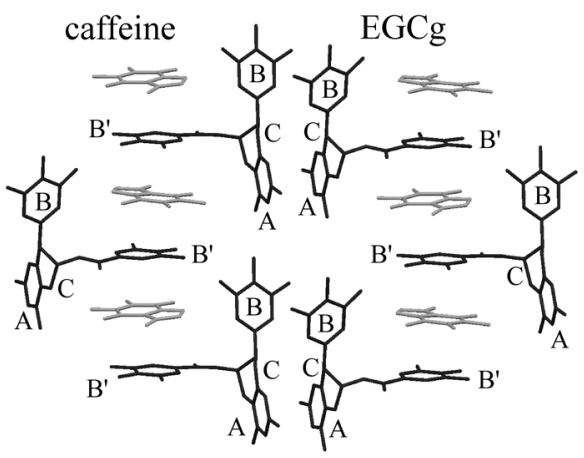

(b)

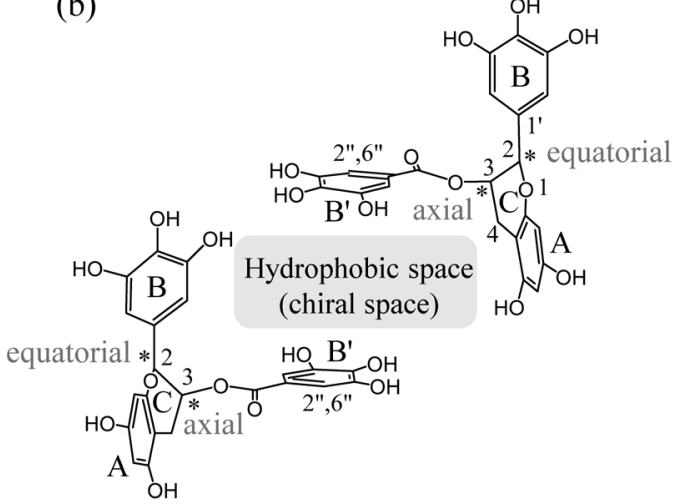

(c)<smiles>O=C1NCC(=O)N2CCC[C@H]12</smiles>

cyclo(Pro-Gly)<smiles>CC(C)NC[C@@H](O)COc1cccc2ccccc12</smiles>

Fig. 1. (a) Layer Structure of the Complex of (-)-Epigallocatechin-3-O-gallate (EGCg) and Caffeine (b) Hydrophobic Space Formed by the A, B, B' Rings of EGCg (c) Cyclo(Pro-Gly) and Propranolol 
Thus, diketopiperazine cyclo(Pro-Gly) was selected as a chiral compound because the molecular size of cyclo(Pro-Gly) was about the same as that of caffeine ${ }^{3)}$ (Fig. 1c). Subsequently, the chiral recognition of diketopiperazines cyclo(L-Pro-Gly) and cyclo(D-Pro-Gly) by EGCg was investigated using several NMR techniques and X-ray crystallographic analysis. ${ }^{4)}$

Therefore, as an application in medicine of the chiral recognition of EGCg, a sympathetic $\beta$-receptor blocker propranolol used for the treatment of hypertension as an ordinary racemic form was investigated (Fig. 1c).

\section{Experimental}

Materials EGCg (>90\%) and (R)-propranolol, $(S)$ propranolol were purchased from Funakoshi Co., Ltd. and Sigma-Aldrich Co., respectively. EGCg was used with further purification.

NMR Experiments ${ }^{1} \mathrm{H}-\mathrm{NMR}$ spectra were recorded at room temperature on a JEOL JMN-LA500 (Tokyo, Japan) operating at $500 \mathrm{MHz} . \mathrm{D}_{2} \mathrm{O}$ was used as a solvent (99.9 atom \% D; Wako Pure Chemical Industries, Ltd., Osaka, Japan). Chemical shift values are expressed in ppm downfield using sodium 2,2-dimethyl-2-silapentane-5-sulfonate (DSS) as an internal standard. The nuclear Overhauser effect (NOE) difference experiments were typically conducted at $35^{\circ} \mathrm{C}$ with $32 \mathrm{~K}$ data points covering a spectral width of $10000 \mathrm{~Hz}$ and with $\mathrm{ca}$. $5 \mathrm{~s}$ presaturation time.

Preparation of Creaming Precipitate Made from EGCg and Cyclo(Pro-Gly) A solution of cyclo(L-Pro-Gly) (1.68 $\left.\mathrm{mg}, 1.09 \times 10^{-2} \mathrm{mmol}\right)$ in $\mathrm{H}_{2} \mathrm{O}(40 \mu \mathrm{L})$ was added to a solution of EGCg $\left(5.00 \mathrm{mg}, 1.09 \times 10^{-2} \mathrm{mmol}\right)$ in $\mathrm{H}_{2} \mathrm{O}(30 \mu \mathrm{L})$. After leaving the mixture at room temperature for $1 \mathrm{~h}$, this was left at $10^{\circ} \mathrm{C}$ for about $24 \mathrm{~h}$ to afford a colorless block crystal $(0.15 \times 0.11 \times 0.06 \mathrm{~mm})$. A solution of cyclo(D-Pro-Gly) (1.68 mg, $\left.1.09 \times 10^{-2} \mathrm{mmol}\right)$ in $\mathrm{H}_{2} \mathrm{O}(40 \mu \mathrm{L})$ was added to a solution of EGCg $\left(5.00 \mathrm{mg}, 1.09 \times 10^{-2} \mathrm{mmol}\right)$ in $\mathrm{H}_{2} \mathrm{O}(30 \mu \mathrm{L})$. By the same procedure as above, the mixture afforded a colorless block crystal $(0.17 \times 0.10 \times 0.04 \mathrm{~mm})$.

X-Ray Crystal Structure Analysis of the 2:2 Complex of EGCg and Cyclo(L-Pro-Gly) A crystal of the $2: 2$ com- plex of EGCg and cyclo(L-Pro-Gly) was determined by X-ray crystallographic analysis at $213 \mathrm{~K}$. The X-ray intensity data of 14640 reflections (of which 8113 were unique) were collected on a Rigaku RAXIS RAPID II imaging plate area detector with graphite monochromated $\mathrm{CuK \alpha}$ radiation $(\lambda=1.54187 \AA)$. The data were corrected for Lorentz and polarization effects. The structure was solved by direct methods using SIR2008 ${ }^{5)}$ and expanded using Fourier techniques." ${ }^{6}$ The final cycle of full-matrix least-squares refinement on $F^{2}$ was based on 8113 observed reflections and 851 variable parameters and converged with unweighted and weighted agreement factors of $R=\sum \| F_{\mathrm{o}}|-| F_{\mathrm{c}}|/| \Sigma\left|F_{\mathrm{o}}\right|=0.0753 \quad(\mathrm{I}>2.00 \sigma(I)), R w=\left[\Sigma\left(w\left(F_{\mathrm{o}}{ }^{2}-\right.\right.\right.$ $\left.\left.\left.F_{\mathrm{c}}^{2}\right)^{2}\right) / \sum w\left(F_{\mathrm{o}}{ }^{2}\right)^{2}\right]^{1 / 2}=0.2288$. The goodness of fit was 1.11 . Unit weights were used. The maximum and minimum peaks on the final difference Fourier map corresponded to 0.33 and $-0.38 \mathrm{e} /$ $\AA^{3}$, respectively. The final Flack parameter was $0.2(4) .^{7)}$ All calculations were performed using the CrystalStructure ${ }^{8)}$ crystallographic software package except for refinement, which was performed using SHELXL-97. $\left.{ }^{9}\right)$ Crystallographic data reported in this manuscript have been deposited with the Cambridge Crystallographic Data Center as supplementary publication No. 1017644 for the 2:2 complex of EGCg and cyclo(L-Pro-Gly).

X-Ray Crystal Structure Analysis of the 2:2 Complex of EGCg and Cyclo(D-Pro-Gly) A crystal of the $2: 2$ complex of EGCg and cyclo(D-Pro-Gly) was determined by X-ray crystallographic analysis at $213 \mathrm{~K}$. X-Ray intensity data of 14231 reflections (of which 7918 were unique) were collected on a Rigaku RAXIS RAPID II imaging plate area detector with graphite monochromated $\mathrm{CuK \alpha}$ radiation $(\lambda=1.54187 \AA)$. The data were corrected for Lorentz and polarization effects. The structure was solved by direct methods using SIR2008 ${ }^{5}$ and expanded using Fourier techniques. ${ }^{6}$ The final cycle of full-matrix least-squares refinement on $F^{2}$ was based on 7918 observed reflections and 856 variable parameters and converged with unweighted and weighted agreement factors of $R=\sum \| F_{\mathrm{o}}|-| F_{\mathrm{c}}|| / \Sigma\left|F_{\mathrm{o}}\right|=0.1138 \quad(I>2.00 \sigma(I)), R w=\left[\Sigma\left(w\left(F_{\mathrm{o}}^{2}-\right.\right.\right.$ $\left.\left.\left.F_{\mathrm{c}}{ }^{2}\right)^{2}\right) / \Sigma w\left(F_{\mathrm{o}}{ }^{2}\right)^{2}\right]^{1 / 2}=0.3551$. The goodness of fit was 1.13 . Unit weights were used. The maximum and minimum peaks on the
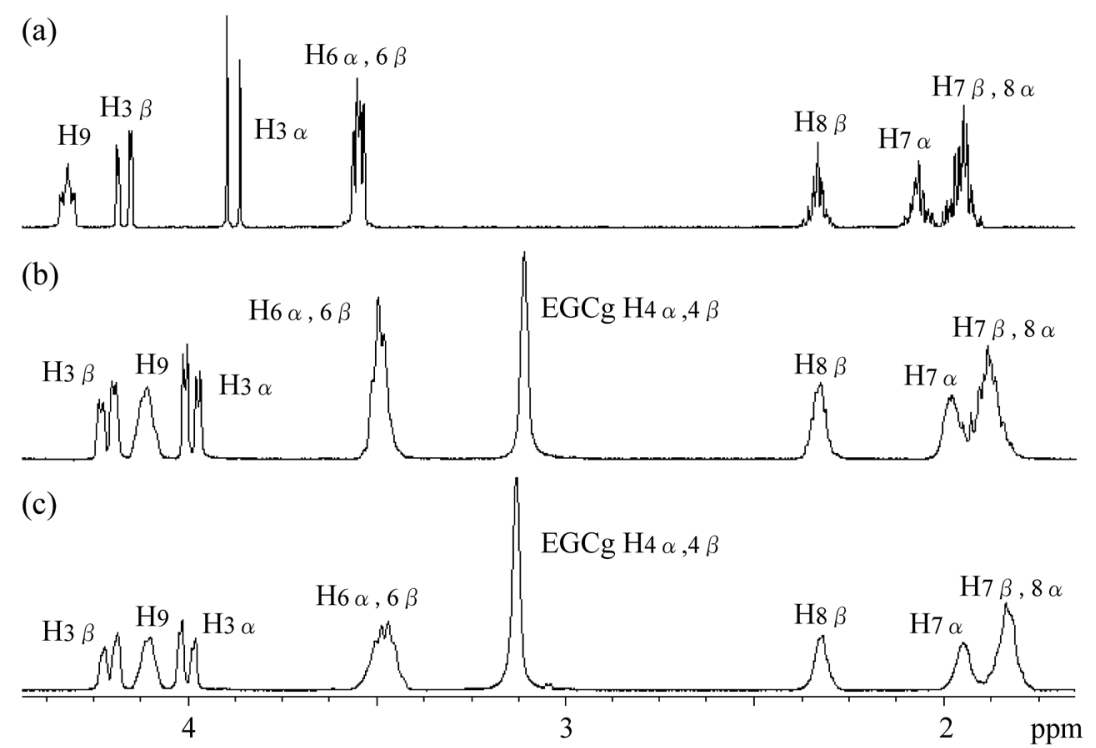

Fig. 2. ${ }^{1} \mathrm{H}-\mathrm{NMR}$ Spectra of a $\mathrm{D}_{2} \mathrm{O}$ Solution Containing (a) Cyclo(L-Pro-Gly) or Cyclo(D-Pro-Gly) (155.8 mu) (b) EGCg and Cyclo(L-Pro-Gly) (Each $155.8 \mathrm{~mm}$ ) in $\mathrm{D}_{2} \mathrm{O}$, and (c) EGCg and Cyclo(D-Pro-Gly) (Each $\left.155.8 \mathrm{~mm}\right)$ 
final difference Fourier map corresponded to 0.42 and -0.37 $\mathrm{e} / \AA^{3}$, respectively. The final Flack parameter was $0.1(4) .^{7)}$ All calculations were performed using the CrystalStructure ${ }^{8)}$ crystallographic software package except for refinement, which was performed using SHELXL-97.9) Crystallographic data reported in this manuscript have been deposited with the Cambridge Crystallographic Data Center as supplementary publication No. 1017643 for the 2:2 complex of EGCg and cyclo(D-Pro-Gly).

Stoichiometry and Thermodynamic Parameters of EGCg Complexes with Cyclo(L-Pro-Gly) and Cyclo(D-Pro-Gly) in the $\mathrm{D}_{2} \mathrm{O}$ Solution The stoichiometry and stability constants $K_{\mathrm{c}}$ of the EGCg complexes with cyclo(L-Pro-Gly) and cyclo(DPro-Gly) were determined by monitoring the chemical shifts of cyclo(L-Pro-Gly) and cyclo(D-Pro-Gly) $\mathrm{H}_{9}$ proton signals in the ${ }^{1} \mathrm{H}-\mathrm{NMR}$ measurements when the concentration of EGCg was continuously increased from 0 to $54 \mathrm{~mm}$ in a constant concentration of cyclo(L-Pro-Gly) and cyclo(D-Pro-Gly) $(10 \mathrm{~mm})$ and using the equation Eq. 1 in the range $40-70^{\circ} \mathrm{C}$.

$$
\begin{aligned}
& \mathrm{A}+n \mathrm{~B} \stackrel{K_{\mathrm{c}}}{\rightleftharpoons} \mathrm{CX} \\
& {[\mathrm{B}]=\left[\frac{1}{\left(\frac{\Delta \delta_{\mathrm{CX}}}{\Delta \delta_{\mathrm{obs}}}-1\right) K_{\mathrm{c}}}\right]^{1 / n}+n \frac{\Delta \delta_{\mathrm{obs}}}{\Delta \delta_{\mathrm{CX}}}[\mathrm{A}]_{0}}
\end{aligned}
$$

Also, the changes in free energy $\Delta G$, enthalpy $\Delta H$, entropy $\Delta S$ for formation of EGCg complexes with cyclo(L-Pro-Gly) and cyclo(D-Pro-Gly) on temperature were estimated by using the results of stability constants. Here, A, B and CX mean cyclo(L-Pro-Gly) or cyclo(D-Pro-Gly), EGCg and complexes of EGCg and cyclo(L-Pro-Gly) or cyclo(D-Pro-Gly), respectively, and $\Delta \delta_{\text {obs }}$ and $\Delta \delta_{\mathrm{CX}}$ represent $\left(\delta_{\mathrm{A}}-\delta_{\text {obs }}\right)$ and $\left(\delta_{\mathrm{A}}-\delta_{\mathrm{CX}}\right)$, respectively. $\delta_{\mathrm{A}}, \delta_{\mathrm{CX}}$, and $\delta_{\mathrm{obs}}$ represent the chemical shift (ppm) of the $\mathrm{H}_{9}$ proton of cyclo(L-Pro-Gly) or cyclo(D-Pro-Gly) in a free state, complexes of EGCg and cyclo(L-Pro-Gly) or cyclo(DPro-Gly), and the mixture of EGCg and cyclo(L-Pro-Gly) or cyclo(D-Pro-Gly) in ${ }^{1} \mathrm{H}-\mathrm{NMR}$ spectra, respectively.

Stoichiometry and Thermodynamic Parameters of EGCg Complexes with $(R)$-Propranolol and $(S)$-Propranolol in the $\mathbf{D}_{2} \mathbf{O}$ Solution The stoichiometry and stability constants $K_{\mathrm{c}}$ of EGCg complexes with $(R)$-propranolol and $(S)$-propranolol were determined by monitoring the chemical shifts of EGCg $\mathrm{H}_{2^{\prime \prime}, 6 "}$ proton signals in the ${ }^{1} \mathrm{H}-\mathrm{NMR}$ measurements when the concentration of $(R)$-propranolol and $(S)$-propranolol were continuously increased from 0 to $54 \mathrm{~mm}$ in a constant concentration of EGCg $(10 \mathrm{~mm})$ and using the equation of Eq. 1 in the range $35-70^{\circ} \mathrm{C}$. Also, the changes in free energy $\Delta G$, enthalpy $\Delta H$, entropy $\Delta S$ for formation of EGCg complexes with $(R)$ propranolol and $(S)$-propranolol in temperature were estimated by using the results of stability constants. Here, A, B and CX mean EGCg, $(R)$-propranolol or $(S)$-propranolol and complexes of EGCg and $(R)$-propranolol or $(S)$-propranolol, respectively, and $\Delta \delta_{\text {obs }}$ and $\Delta \delta_{\mathrm{CX}}$ represent $\left(\delta_{\mathrm{A}}-\delta_{\text {obs }}\right)$ and $\left(\delta_{\mathrm{A}}-\delta_{\mathrm{CX}}\right)$, respectively. $\delta_{\mathrm{A}}, \delta_{\mathrm{CX}}$, and $\delta_{\mathrm{obs}}$ represent the chemical shift (ppm) of the $\mathrm{H}_{2 ", 6 "}$ proton of EGCg in a free state, complexes of EGCg and $(R)$-propranolol or $(S)$-propranolol, and the mixture of EGCg and $(R)$-propranolol or $(S)$-propranolol in ${ }^{1} \mathrm{H}-\mathrm{NMR}$ spectra, respectively.

\section{Results and Discussion}

NMR Study of Complex of EGCg and Diketopiperazine Cyclo(Pro-Gly) A solution of diketopiperazines cyclo(LPro-Gly), cyclo(D-Pro-Gly) in $\mathrm{D}_{2} \mathrm{O}$ was added to a solution of an equimolecular amount of EGCg in $\mathrm{D}_{2} \mathrm{O}$. ${ }^{1} \mathrm{H}-\mathrm{NMR}$ spectra of the mixture are shown in Figs. $2 \mathrm{~b}$ and $\mathrm{c}$. All proton signals derived from cyclo(L-Pro-Gly) and cyclo(D-Pro-Gly) appeared as broad signals, as compared with the corresponding proton signals of the ${ }^{1} \mathrm{H}-\mathrm{NMR}$ spectra of cyclo(L-Pro-Gly), and cyclo(D-Pro-Gly) alone in $\mathrm{D}_{2} \mathrm{O}$ (Fig. 2a). It was thought that cyclo(L-Pro-Gly), cyclo(D-Pro-Gly) formed complexes with $\mathrm{EGCg}$, and that the motion of their protons was restricted, leading to their signals being broadened.

Table 1 shows the chemical shift of ${ }^{1} \mathrm{H}-\mathrm{NMR}$ signals of a solution containing equimolecular amounts of $\mathrm{EGCg}$ and cyclo(L-Pro-Gly), cyclo(D-Pro-Gly) in $\mathrm{D}_{2} \mathrm{O}$, and indicates the shift values started from the chemical shift of ${ }^{1} \mathrm{H}-\mathrm{NMR}$ signals of a solution containing cyclo(L-Pro-Gly) and position of

Table 1. Chemical Shift of ${ }^{1} \mathrm{H}-\mathrm{NMR}$ Signals in a Solution Containing Equimolecular Amounts of EGCg and Cyclo(L-Pro-Gly), Cyclo(D-Pro-Gly) in $\mathrm{D}_{2} \mathrm{O}$ (Each $155.8 \mathrm{~mm}$ )

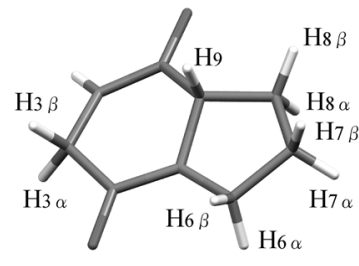

cyclo(L-Pro-Gly)

\begin{tabular}{lccc}
\hline \hline \multicolumn{4}{c}{ Chemical shift (ppm) of cyclo(L-Pro-Gly) } \\
\hline Proton & $\delta(\mathrm{ppm})$ & $\delta(\mathrm{ppm})$ in the presence \\
of EGCg & $\begin{array}{c}\text { Shift value } \\
(\mathrm{ppm})\end{array}$ \\
\hline 9 & 4.319 & 4.108 & -0.211 \\
$3 \beta$ & 4.170 & 4.215 & +0.045 \\
$3 \alpha$ & 3.881 & 3.989 & +0.108 \\
$6 \alpha, \beta$ & 3.549 & 3.497 & -0.052 \\
$8 \beta$ & 2.332 & 2.325 & -0.007 \\
$7 \alpha$ & 2.064 & 1.977 & -0.087 \\
$7 \beta, 8 \alpha$ & 1.947 & 1.882 & -0.065 \\
\hline
\end{tabular}

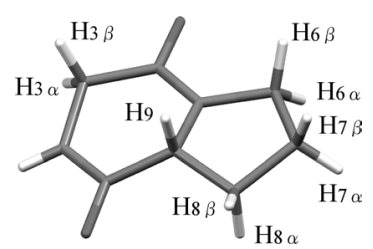

cyclo(D-Pro-Gly)

\begin{tabular}{lccc}
\hline \hline \multicolumn{4}{c}{ Chemical shift (ppm) of cyclo(D-Pro-Gly) } \\
\hline Proton & $\delta(\mathrm{ppm})$ & $\begin{array}{c}\delta(\mathrm{ppm}) \text { in the presence } \\
\text { of EGCg }\end{array}$ & $\begin{array}{c}\text { Shift value } \\
(\mathrm{ppm})\end{array}$ \\
\hline 9 & 4.320 & 4.100 & -0.220 \\
$3 \beta$ & 4.170 & 4.207 & +0.037 \\
$3 \alpha$ & 3.880 & 4.001 & +0.121 \\
$6 \alpha, \beta$ & 3.548 & 3.480 & -0.068 \\
$8 \beta$ & 2.333 & 2.319 & -0.014 \\
$7 \alpha$ & 2.065 & 1.947 & -0.118 \\
$7 \beta, 8 \alpha$ & 1.947 & 1.834 & -0.113 \\
\hline
\end{tabular}


Table 2. The Stability Constants $K_{\mathrm{c}}$, the Changes in Free Energy $\Delta G$, Enthalpy $\Delta H$, Entropy $\Delta S$ and the Order of the Reaction $n$ of EGCg Complexes with Cyclo(L-Pro-Gly) and Cyclo(D-Pro-Gly)

\begin{tabular}{|c|c|c|c|c|c|}
\hline \multicolumn{6}{|c|}{ EGCg-cyclo(L-Pro-Gly) } \\
\hline$T\left({ }^{\circ} \mathrm{C}\right)$ & $K_{\mathrm{c}}\left(\mathrm{M}^{-1}\right)$ & $\Delta G\left(\mathrm{~kJ} \mathrm{~mol}^{-1}\right)$ & $\Delta H\left(\mathrm{~kJ} \mathrm{~mol}^{-1}\right)$ & $\Delta S\left(\mathrm{~J} \mathrm{~mol}^{-1} \mathrm{~K}^{-1}\right)$ & $n$ \\
\hline 40 & 996.3 & -18.0 & \multirow{6}{*}{-31.8} & \multirow{6}{*}{-44.1} & 0.81 \\
\hline 45 & 846.9 & -17.8 & & & 0.89 \\
\hline 50 & 716.7 & -17.7 & & & 0.91 \\
\hline 55 & 583.7 & -17.4 & & & 1.05 \\
\hline 60 & 479.5 & -17.1 & & & 1.24 \\
\hline 70 & 348.7 & -16.7 & & & 1.34 \\
\hline \multicolumn{6}{|c|}{ EGCg-cyclo(D-Pro-Gly) } \\
\hline$T\left({ }^{\circ} \mathrm{C}\right)$ & $K_{\mathrm{c}}\left(\mathrm{M}^{-1}\right)$ & $\Delta G\left(\mathrm{~kJ} \mathrm{~mol}^{-1}\right)$ & $\Delta H\left(\mathrm{~kJ} \mathrm{~mol}^{-1}\right)$ & $\Delta S\left(\mathrm{~J} \mathrm{~mol}^{-1} \mathrm{~K}^{-1}\right)$ & $n$ \\
\hline 40 & 1072.1 & -18.2 & \multirow{6}{*}{-31.7} & \multirow{6}{*}{-43.8} & 0.83 \\
\hline 45 & 762.2 & -17.5 & & & 0.93 \\
\hline 50 & 659.8 & -17.4 & & & 0.98 \\
\hline 55 & 570.0 & -17.3 & & & 1.01 \\
\hline 60 & 477.8 & -17.1 & & & 1.24 \\
\hline 70 & 350.0 & -16.9 & & & 1.05 \\
\hline
\end{tabular}

(a)

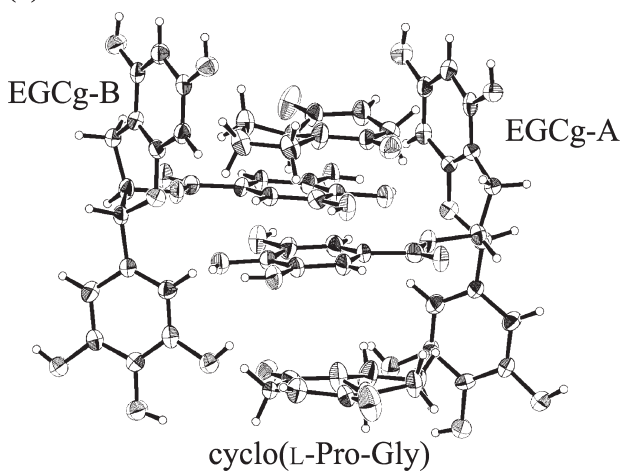

(b)

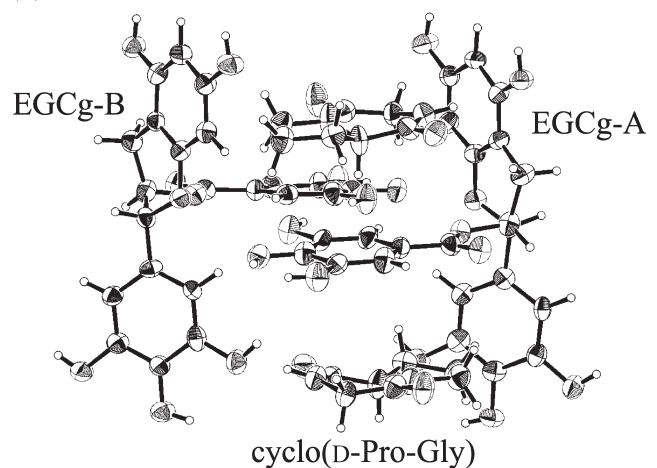

Fig. 3. ORTEP Drawings with Thermal Ellipsoids at a 30\% Probability Level

(a) 2:2 complex of EGCg and cyclo(L-Pro-Gly) (b) 2:2 complex of EGCg and cyclo(D-Pro-Gly).

(a)

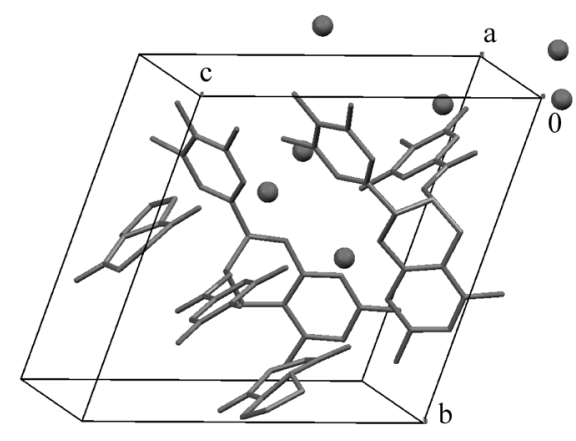

(b)

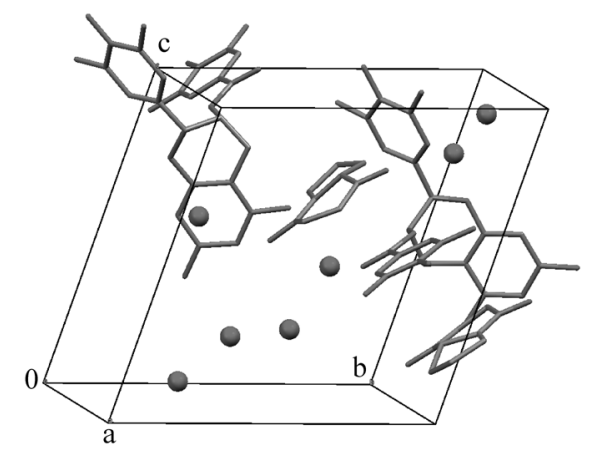

Fig. 4. One Unit Cell

Hydrogen atoms are omitted for clarity. (a) 2:2 complex of EGCg and cyclo(L-Pro-Gly) (b) 2:2 complex of EGCg and cyclo(D-Pro-Gly).

the Pro residue of cyclo(L-Pro-Gly) and cyclo(D-Pro-Gly) was observed, while a downfield shift of the proton signal $\mathrm{H}_{3 \alpha}$ in the $\alpha$ position of the Gly residue was also observed. Upfield shifts in proton signals $\mathrm{H}_{7 \alpha, \beta}$ and $\mathrm{H}_{8 \alpha}$ in the $\beta$ and $\gamma$ positions of the Pro residue of cyclo(D-Pro-Gly) were more marked than those of cyclo(L-Pro-Gly).
Thermodynamic data on the complex formation of EGCg and cyclo(L-Pro-Gly), cyclo(D-Pro-Gly) were investigated. The stability constants $K_{\mathrm{c}}$ of the complexes of EGCg and cyclo(LPro-Gly), cyclo(D-Pro-Gly) at $40^{\circ} \mathrm{C}$ were 996.3 and $1072.1 \mathrm{~m}^{-1}$, respectively (Table 2 ).

From the dependency of $K_{\mathrm{c}}$ on temperature, the change in 
Table 3. Torsion Angles of EGCg Moieties of 2:2 EGCg Complexes with Cyclo(L-Pro-Gly) and Cyclo(D-Pro-Gly)

\begin{tabular}{cccccc}
\hline \hline \multirow{2}{*}{ Torsion angle } & \multicolumn{2}{c}{ EGCg in cyclo(L-Pro-Gly) complex } & \multicolumn{2}{c}{ EGCg in cyclo(D-Pro-Gly) complex } \\
\cline { 2 - 5 } & $\mathrm{A}$ & $169^{\circ}$ & $168^{\circ}$ & A & $169^{\circ}$ \\
\hline$\angle \mathrm{H}_{2}-\mathrm{C}_{2}-\mathrm{C}_{3}-3 \mathrm{O}$ & $172.0(6)^{\circ}$ & $173.6(6)^{\circ}$ & & $171.8(7)^{\circ}$ & $170^{\circ}$ \\
$\angle \mathrm{C}_{1},-\mathrm{C}_{2}-\mathrm{C}_{3}-\mathrm{C}_{4}$ & $173.7(6)^{\circ}$ & $173.7(6)^{\circ}$ & & $174.1(7)^{\circ}$ & $169.1(8)^{\circ}$ \\
$\angle \mathrm{C}_{1},-\mathrm{C}_{2}-\mathrm{C}_{1}-\mathrm{C}_{8 \mathrm{a}}$ & & & & $176.0(7)^{\circ}$ \\
\hline
\end{tabular}
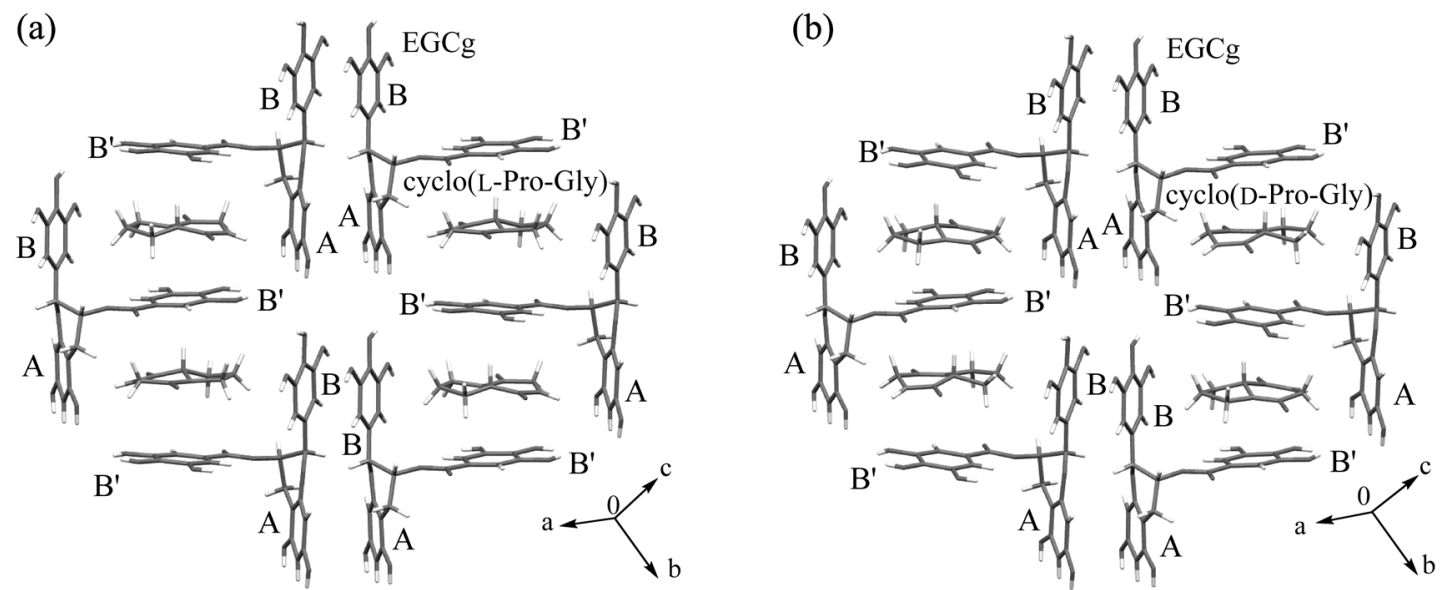

Fig. 5. Layer Structures (a) 2:2 Complex of EGCg and Cyclo(L-Pro-Gly) (b) 2:2 Complex of EGCg and Cyclo(D-Pro-Gly)
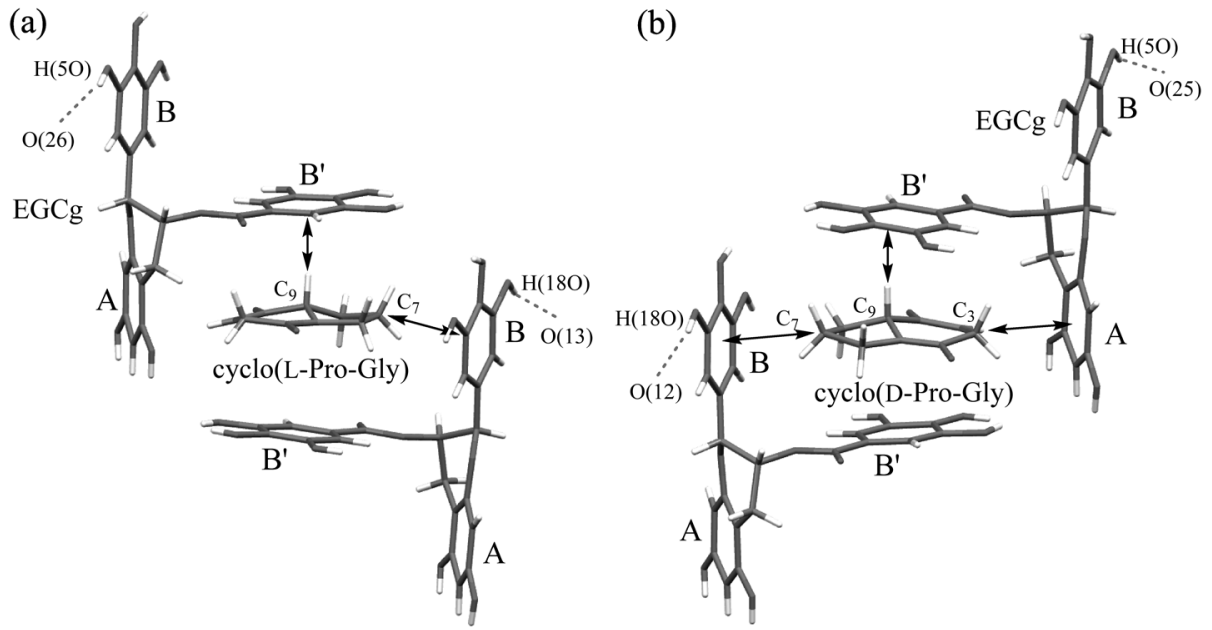

Fig. 6. Intermolecular Interactions

(a) 2:2 complex of EGCg and cyclo(L-Pro-Gly) (b) 2:2 complex of EGCg and cyclo(D-Pro-Gly). Black arrows and dotted lines indicate CH- $\pi$ interactions and hydrogen bonds, respectively.

free energy $\Delta G$, enthalpy $\Delta H$ and entropy $\Delta S$ of the complex formation were estimated as shown in Table 2. The thermodynamic data for the complex formation of EGCg and cyclo(LPro-Gly) were very similar to those of EGCg and cyclo(DPro-Gly). The entropy $(\Delta S)$ for the formation of the complex of EGCg and cyclo(L-Pro-Gly), cyclo(D-Pro-Gly) had large negative values of -44.1 and $-43.8 \mathrm{~J} \mathrm{~mol}^{-1} \mathrm{~K}^{-1}$, respectively, suggesting that cyclo(L-Pro-Gly) and cyclo(D-Pro-Gly) were fixed tightly in the complex with EGCg.

There is no great difference between the thermodynamic data of the complex formation of EGCg with cyclo(L-Pro-Gly) and those with cyclo(D-Pro-Gly), indicating that the physical properties of the complex of EGCg with cyclo(L-Pro-Gly) and those with cyclo(D-Pro-Gly) are very similar.
Determination of the Stereochemical Structure of the Complex of EGCg and Cyclo(Pro-Gly) A solution of diketopiperazine cyclo(L-Pro-Gly) in $\mathrm{H}_{2} \mathrm{O}$ was added to a solution of an equimolecular amount of EGCg in $\mathrm{H}_{2} \mathrm{O}$. The mixture afforded a colorless block crystal, which contained EGCg and cyclo(L-Pro-Gly) at a molar ratio of $1: 1$, based on measurement of the integral volume of ${ }^{1} \mathrm{H}-\mathrm{NMR}$ signals. A single crystal was determined to be a $2: 2$ complex of EGCg and cyclo(L-Pro-Gly) by X-ray crystallographic analysis. ${ }^{3)}$

Using the same method as for crystallization of the complex of EGCg and cyclo(D-Pro-Gly), a single crystal of a complex of EGCg and cyclo(D-Pro-Gly) was prepared and determined to be a 2:2 complex of EGCg and cyclo(D-Pro-Gly) by X-ray crystallographic analysis. ${ }^{3)}$ 
<smiles>CC(C)NC[C@H](O)COc1cccc2ccccc12</smiles>

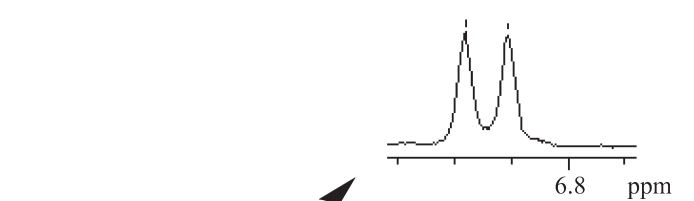

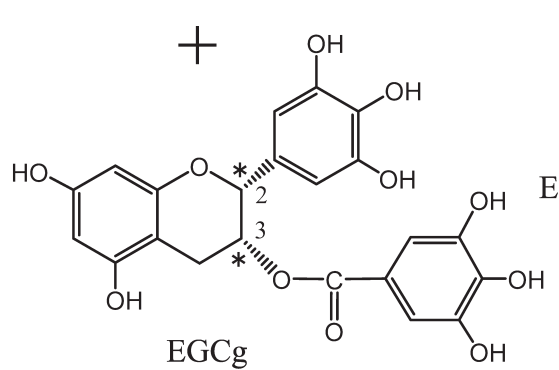

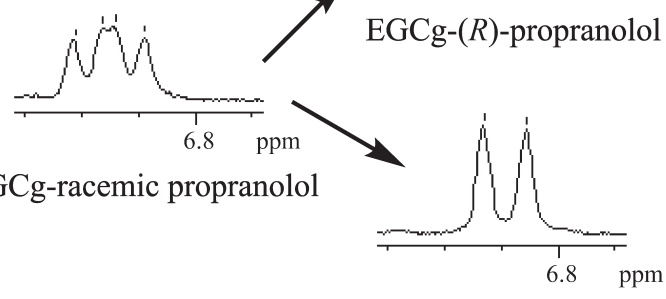

EGCg-(S)-propranolol

Fig. 7. Separation of the Racemic Propranolol $\mathrm{H}_{2}$ Proton Signal

Measurement condition of ${ }^{1} \mathrm{H}-\mathrm{NMR}$ spectra: EGCg and racemic propranolol (each $40 \mathrm{~mm}$ ) in $\mathrm{D}_{2} \mathrm{O}$ at room temperature.

(a)

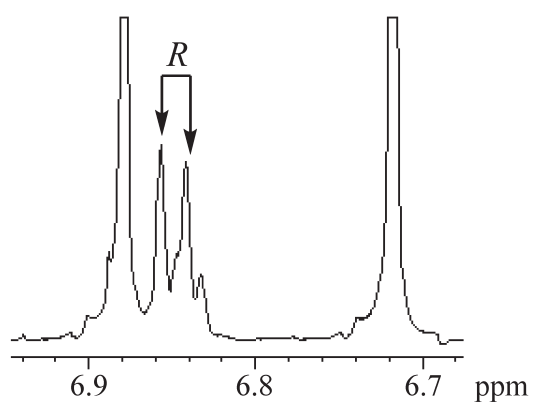

(b)

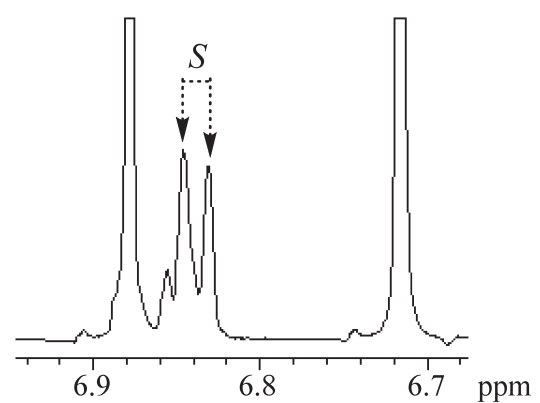

(c)

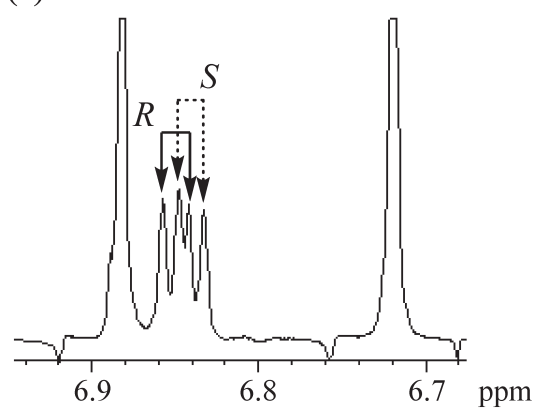

Fig. 8. Assignment of $(R)$ - and $(S)$-Propranolol $\mathrm{H}_{2}$ Proton Signals in the Presence of EGCg $(40 \mathrm{~mm})$

Concentration of $(R)$ - and (S)-propranolol: (a) 30 and $10 \mathrm{~mm}$, (b) 10 and $30 \mathrm{~mm}$, (c) 20 and $20 \mathrm{~mm}$.

Table 4. The Stability Constants $K_{\mathrm{c}}$, the Changes in Free Energy $\Delta G$, Enthalpy $\Delta H$, Entropy $\Delta S$ and the Order of the Reaction $n$ of EGCg Complexes with $(R)$ - and $(S)$-Propranolol

\begin{tabular}{|c|c|c|c|c|c|}
\hline \multicolumn{6}{|c|}{ EGCg- $(R)$-propranolol } \\
\hline$T\left({ }^{\circ} \mathrm{C}\right)$ & $K_{\mathrm{c}}\left(\mathrm{M}^{-1}\right)$ & $\Delta G\left(\mathrm{~kJ} \mathrm{~mol}^{-1}\right)$ & $\Delta H\left(\mathrm{~kJ} \mathrm{~mol}^{-1}\right)$ & $\Delta S\left(\mathrm{~J} \mathrm{~mol}^{-1} \mathrm{~K}^{-1}\right)$ & $n$ \\
\hline 35 & 943.7 & -17.5 & \multirow{7}{*}{-24.4} & \multirow{7}{*}{-21.7} & 0.86 \\
\hline 40 & 835.2 & -17.5 & & & 0.87 \\
\hline 45 & 893.8 & -18.0 & & & 0.89 \\
\hline 50 & 664.9 & -17.5 & & & 0.89 \\
\hline 55 & 579.6 & -17.3 & & & 0.91 \\
\hline 60 & 490.8 & -17.2 & & & 0.91 \\
\hline 70 & 369.5 & -16.9 & & & 0.93 \\
\hline \multicolumn{6}{|c|}{ EGCg-(S)-propranolol } \\
\hline$T\left({ }^{\circ} \mathrm{C}\right)$ & $K_{\mathrm{c}}\left(\mathrm{M}^{-1}\right)$ & $\Delta G\left(\mathrm{~kJ} \mathrm{~mol}^{-1}\right)$ & $\Delta H\left(\mathrm{~kJ} \mathrm{~mol}^{-1}\right)$ & $\Delta S\left(\mathrm{~J} \mathrm{~mol}^{-1} \mathrm{~K}^{-1}\right)$ & $n$ \\
\hline 35 & 871.8 & -17.3 & & & 0.87 \\
\hline 40 & 838.1 & -17.5 & & & 0.89 \\
\hline 45 & 793.2 & -17.7 & & & 0.91 \\
\hline 50 & 699.1 & -17.6 & -19.6 & -6.7 & 0.92 \\
\hline 55 & 608.4 & -17.5 & & & 0.91 \\
\hline 60 & 495.0 & -17.2 & & & 0.94 \\
\hline 70 & 424.8 & -17.3 & & & 1.00 \\
\hline
\end{tabular}

ORTEP drawings of 2:2 complexes of EGCg and cyclo(LPro-Gly), EGCg and cyclo(D-Pro-Gly) are shown in Figs. 3a and $b$. The $2: 2$ complexes were formed from two crystallo- graphically different EGCgs (EGCg-A and EGCg-B) and two cyclo(L-Pro-Gly)s, two cyclo(D-Pro-Gly)s.

One unit cell contained one unit of the $2: 2$ complex of 
EGCg and cyclo(L-Pro-Gly), cyclo(D-Pro-Gly) and seven water molecules as a crystal solvent (Figs. $4 \mathrm{a}, \mathrm{b}$ ).

The torsion angles of EGCg moieties (EGCg-A and EGCgB) of the 2:2 complexes of EGCg and cyclo(L-Pro-Gly), cyclo(D-Pro-Gly) indicated that the B rings of EGCg-A and EGCg-B were both at equatorial positions, while the $\mathrm{B}^{\prime}$ rings of EGCg-A and EGCg-B were both in axial positions with respect to the $\mathrm{C}$ rings of the EGCg molecules (Table 3).

In the layer structures of the 2:2 complexes of EGCg and cyclo(L-Pro-Gly), cyclo(D-Pro-Gly), cyclo(L-Pro-Gly) and cyclo(D-Pro-Gly) were captured by the space formed by the three aromatic $\mathrm{A}, \mathrm{B}, \mathrm{B}^{\prime}$ rings of $\mathrm{EGCg}$ and were located almost in the middle of the two $\mathrm{B}^{\prime}$ rings of EGCg-A or EGCg$\mathrm{B}$ (Figs. 5a, b), the same as caffeine in the $2: 2$ complex of EGCg (Fig. 1a).

In the 2:2 complexes of EGCg and cyclo(L-Pro-Gly), cyclo(D-Pro-Gly), intermolecular interactions forming between EGCg and cyclo(L-Pro-Gly), cyclo(D-Pro-Gly) were elucidated. In the $2: 2$ complex of EGCg and cyclo(L-Pro-Gly), $\mathrm{CH}-\pi$ interactions formed between methine $\mathrm{C}_{9}-\mathrm{H}$ of cyclo(L-Pro-Gly)

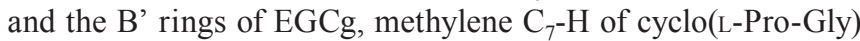
and the $\mathrm{B}$ rings of $\mathrm{EGCg}$, and two $\mathrm{O}-\mathrm{H}$... O intermolecular hydrogen bonds were observed between EGCg and cyclo(LPro-Gly) (Fig. 6a). In the 2:2 complex of EGCg and cyclo(DPro-Gly), $\mathrm{CH}-\pi$ interactions formed between methine $\mathrm{C}_{9}-\mathrm{H}$ of cyclo(D-Pro-Gly) and the $\mathrm{B}^{\prime}$ rings of $\mathrm{EGCg}$, methylene $\mathrm{C}_{7}-\mathrm{H}$ of cyclo(D-Pro-Gly) and the $\mathrm{B}$ rings of EGCg. In addition, $\mathrm{CH}-\pi$ interactions formed between methylene $\mathrm{C}_{3}-\mathrm{H}$ of
cyclo(D-Pro-Gly) and the A rings of EGCg. Furthermore, in the same way as the $2: 2$ complex of cyclo(L-Pro-Gly), two $\mathrm{O}-\mathrm{H}$...O intermolecular hydrogen bonds were observed (Fig. 6b).

Judging from the crystal structures of 2:2 complexes of EGCg and cyclo(L-Pro-Gly), cyclo(D-Pro-Gly), the upfield shift in the proton signal $\mathrm{H}_{9}$ resulted from magnetic anisotropic shielding by the ring current from the $\mathrm{B}^{\prime}$ ring of the EGCg moieties (Fig. 6). Upon the formation of the 2:2 complex, the upfield shift values of the proton signals $\mathrm{H}_{7 \alpha}, \mathrm{H}_{7 \beta, 8 \alpha}$ of cyclo(LPro-Gly) were 0.087 and $0.065 \mathrm{ppm}$, respectively, and those of cyclo(D-Pro-Gly) were 0.118 and 0.113 ppm, respectively (Table 1). Such a difference between the upfield shift values of the proton signals of cyclo(L-Pro-Gly) and cyclo(D-Pro-Gly) were thought to result mainly from the magnitude of the magnetic anisotropic shielding by the ring current from the B ring of EGCg.

Therefore, it was considered that upon formation of the $2: 2$ complex, a chirality of cyclo(Pro-Gly) was recognized by the magnetic anisotropic shielding effect of the ring current from the $\mathrm{B}$ ring of $\mathrm{EGCg}$.

Chiral Recognition of Propranolol Using EGCg A solution of racemic propranolol $((R)$ - and $(S)$-propranolols) in $\mathrm{D}_{2} \mathrm{O}$ was added to a solution of an equimolecular amount of EGCg in $\mathrm{D}_{2} \mathrm{O}$. In the ${ }^{1} \mathrm{H}-\mathrm{NMR}$ spectrum of the mixture, the $\mathrm{H}_{2}$ proton signal of racemic propranolol was observed as two doublets (Fig. 7), suggesting that $(R)$ - and $(S)$-propranolols each formed complexes with EGCg, which are diastereomers
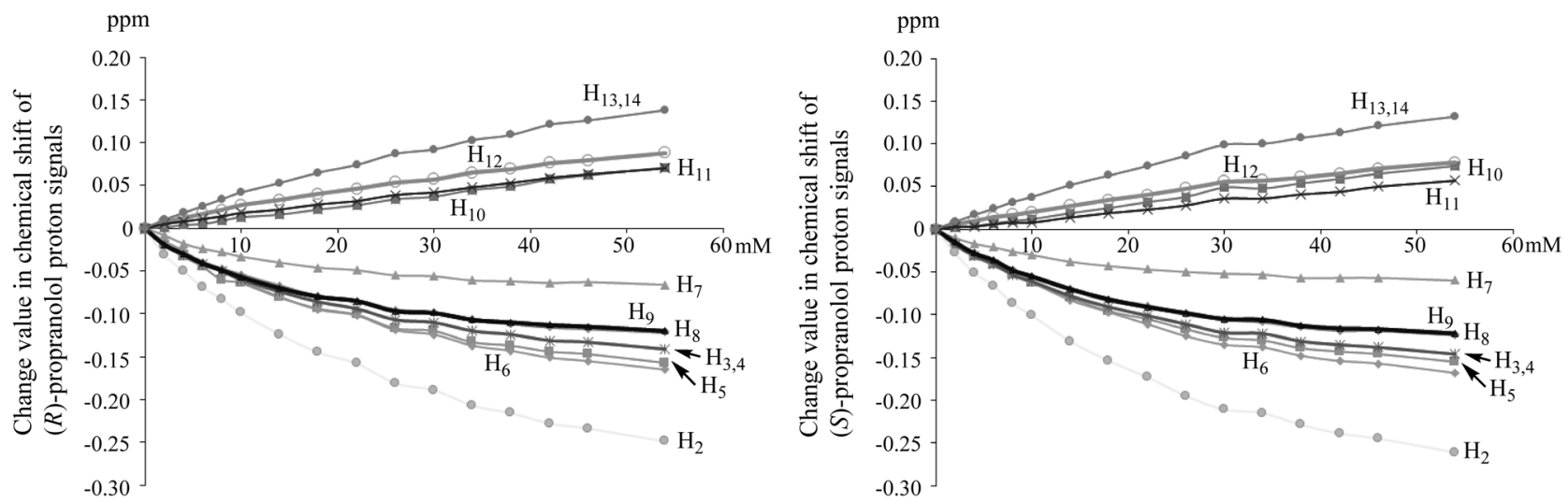

Fig. 9. Change Values in Chemical Shift of $(R)$ - and $(S)$-Propranolol Proton Signals by Increasing EGCg from 0 to $54 \mathrm{~mm}$ (a) $(R)$-Propranolol (b) (S)-Propranolol

Measurement conditions of ${ }^{1} \mathrm{H}-\mathrm{NMR}$ spectra: $(R)$ - and $(S)$-propranolol (each $10 \mathrm{~mm}$ ) in $\mathrm{D}_{2} \mathrm{O}$ at room temperature.

(a)

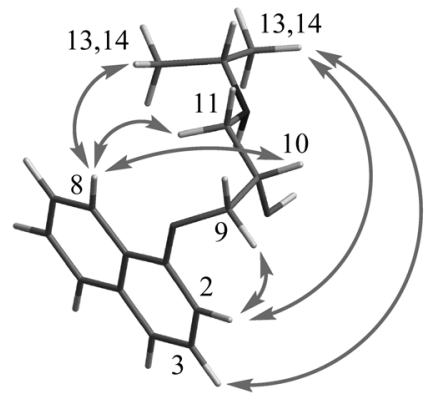

(b)

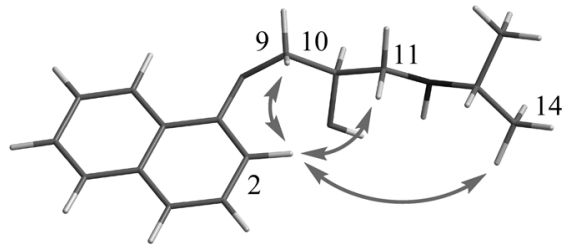

(c)

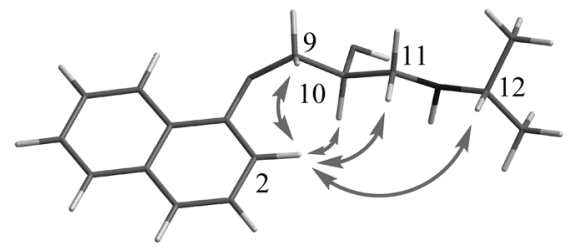

Fig. 10. Intramolecular NOEs (a) (S)-Propranolol Alone (40 mm) (b) (R)-Propranolol (40 mm) in the Presence of EGCg (40 mM) (c) (S)-Propranolol $(40 \mathrm{~mm})$ in the Presence of EGCg $(40 \mathrm{~mm})$

Both arrows indicate intramolecular NOEs. 
of each other. By adding EGCg, the $\mathrm{H}_{2}$ proton signal of $(R)$ and $(S)$-propranolols was observed as two doublets, showing that EGCg recognized the chirality of propranolol.

For assignment of the two doublets, $(R)$ - and $(S)$-propranolols were added to a solution containing an equimolecular amount of EGCg and racemic propranolol in $\mathrm{D}_{2} \mathrm{O}$ (Fig. 8). As a result, doublets of 6.850 and $6.841 \mathrm{ppm}$ were assigned to the $\mathrm{H}_{2}$ of $(R)$ - and $(S)$-propranolols, respectively.

A kinetic on the formation of complexes of $(R)$ - and $(S)$ propranolols and EGCg was performed. The stability constants for the formation of complexes of EGCg and $(R)$-, $(S)$-propranolol $K_{\mathrm{c}}$ at $35-70^{\circ} \mathrm{C}$ were estimated, which assumed the order of the reaction $n$. The reaction $n$ of the complexes of EGCg and $(R)-,(S)$-propranolol at $35^{\circ} \mathrm{C}$ were 0.86 and 0.87 , respectively (Table 4$)$, suggesting that $(R)$ - and $(S)$-propranolol each formed a 1:1 complex of EGCg.

From the dependency of $K_{\mathrm{c}}$ on temperature, the change in free energy $\Delta G$, enthalpy $\Delta H$ and entropy $\Delta S$ of the complex formation were estimated (Table 4). The entropy $(\Delta S)$ for the formation of the complex of EGCg and $(R)$-, $(S)$-propranolols had large negative values of -21.7 and $-6.7 \mathrm{~J} \mathrm{~mol}^{-1} \mathrm{~K}^{-1}$, respectively. It was suggested that $(R)$-propranolol was fixed tightly in the complex of EGCg and $(R)$-propranolol, while $(S)$-propranolol fitted loosely in the complex of EGCg and $(S)$ propranolol.

Next, changes in chemical shifts of proton signals of $(R)$ and $(S)$-propranolols in ${ }^{1} \mathrm{H}-\mathrm{NMR}$ spectra by adding regular amount of EGCg were observed (Fig. 9). Upfield shifts in proton signals for $\mathrm{H}_{9}$ in the side chain and $\mathrm{H}_{2}, \mathrm{H}_{3}, \mathrm{H}_{4}, \mathrm{H}_{5}, \mathrm{H}_{6}, \mathrm{H}_{7}$, $\mathrm{H}_{8}$ in the naphthalene ring of $(R)$ - and $(S)$-propranolols were observed. It was considered that the upfield shifts of proton signals resulted from the magnetic anisotropic shielding by the ring current from the $\mathrm{B}^{\prime}$ ring of EGCg when the naphthalene ring of propranolol was captured by the hydrophobic space formed by EGCg as with the caffeine moieties of the $2: 2$ complex of EGCg and caffeine shown in Fig. 1a.

Upon the complex formation, intermolecular interactions between EGCg and propranolol moieties were investigated. The naphthalene ring of propranolol was considered to form a $\pi-\pi$ interaction with the $\mathrm{B}^{\prime}$ ring of EGCg.

Judging from the entropy $(\Delta S)$ value (Table 4$)$, the complexes of EGCg and $(R)$-propranolol were expected to form not only the $\pi-\pi$ interaction between the naphthalene ring of $(R)$ propranolol and the B' ring of EGCg, but also an intermolecular hydrogen bond between the side chain of $(R)$-propranolol and hydroxyl group of EGCg.

Measurements of the intramolecular nuclear Overhauser effect (NOE) of $(R)$ - and $(S)$-propranolols in the complexes with EGCg in $\mathrm{D}_{2} \mathrm{O}$ were performed (Figs. 10b, c). For a comparison, the intramolecular NOE of $(S)$-propranolol alone in $\mathrm{D}_{2} \mathrm{O}$ was measured (Fig. 10a). A large number of NOEs were observed in $(S)$-propranolol in a free state, but not in $(R)$ and $(S)$-propranolols in the complex with EGCg, suggesting that their conformers were fixed by forming complexes with EGCg.

A characteristic intramolecular NOE in the solution of EGCg and $(S)$-propranolol was observed between $\mathrm{H}_{2}$ in the naphthalene ring and $\mathrm{H}_{10}$ in the side chain of $(S)$-propranolol, but was not observed in the solution of $(R)$-propranolol. It was considered that the $\mathrm{H}_{2}$ proton signal of $(R)$-propranolol was observed in a lower field than that of $(S)$-propranolol due to the influence of the oxygen atom of the hydroxyl group of $(R)$ propranolol in the neighborhood.

It was therefore concluded that a difference in the chemical shift of the $\mathrm{H}_{2}$ proton signal between $(R)$ - and $(S)$-propranolols resulted from a difference in their conformations in the complexes with EGCg, and then EGCg recognized a chirality of racemic propranolol in the $\mathrm{D}_{2} \mathrm{O}$ solution.

Acknowledgment This study was supported by JSPS KAKENHI Grant Number 26860074.

Conflict of Interest The authors declare no conflict of interest.

\section{References and Notes}

1) Maruyama N., Suzuki Y., Sakata K., Yagi A., Ina K., Proc. International Symposium Tea Science, 145-149 (1991).

2) Tsutsumi H., Sato T., Ishizu T., Chem. Lett., 41, 1669-1671 (2012).

3) Preliminary communication Ishizu T., Tsutsumi H., Yokoyama A., Tetrahedron Lett., 56, 1111-1114 (2015).

4) Cyclo(Pro-Gly) was prepared as follows; A mixed anhydride method using Boc-Pro and Gly-OBzl $p$-tosylate by isobutyl chlorocarbonate afforded Boc-Pro-Gly-OBzl, and then a cyclization reaction of Boc-Pro-Gly-OBzl in presence of $N$-methyl-morpholine as a base catalyst afforded cyclo(Pro-Gly) in good yields.

5) SIR2008: Burla M. C., Caliandro R., Camalli M., Carrozzini B., Cascarano G. L., De Caro L., Giacovazzo C., Polidori G., Siliqi D., Spagna R., J. Appl. Cryst., 40, 609-613 (2007).

6) DIRDIF99: Beurskens P. T., Admiraal G., Beurskens G., Bosman W. P., Gelder R. De, Israel R., Smits J. M. M., The DIRDIF-99 program system, Technical Report of the Crystallography Laboratory, University of Nijmegen, the Netherlands, 1999.

7) Flack H. D., Acta Crystallogr. A, 39, 876-881 (1983).

8) CrystalStructure 4.1: Crystal Structure Analysis Package, Rigaku Corporation (2000-2014), Tokyo 196-8666, Japan.

9) Sheldrick G. M., SHELXL97, Acta Crystallogr. A, 64, 112-122 (2008). 